\title{
Neo-Fashion: A Data-Driven Fashion Trend Forecasting System using Machine Learning through Catwalk Analysis
}

\author{
Li Zhao, University of Missouri \\ Muzhen Li, University of Missouri \\ Peng Sun, The Climate Corporation
}

Keywords: Data-Driven Fashion Trends Forecasting; Deep Neural Network; Recommendation System; Catwalk Analysis; Autonomous Prediction

Trend forecasting is crucially important and challenging in the fashion industry (Bikhchandani, S., Hirshleifer, D., \& Welch,1992), and recently has been an emerging research area in computer vision and machine learning (Vittayakorn et al., 2015; Liu et al., 2016; Han et al., 2017). In the fashion world, trend forecasting is defined as the search for a means to predict mood, behavior, and buying habits of the consumer through identifying trends (Halland \& Jones, 2017). With the advent of computational approach, it's possible to translate the creativity and inspiration of practitioners into a data-driven structure, especially for short-term forecasting which is the main focus of this study.

Grounded in theories of fashion innovation, new fashion ideas usually start on designer runways. Fashion ideas follow trickle-down, trickle-across, and trickle-up processes, and last at least through a selling season. The life cycle of a fashion trend can vary, but successful examples last at least one season. Seasonal trends are often catwalk-inspired trends that manifest as key items, colors, silhouettes or ways of styling an outfit. They become the dominating looks during the season, but they drift out of favor after 6-12 months, as consumers move on the next trend.

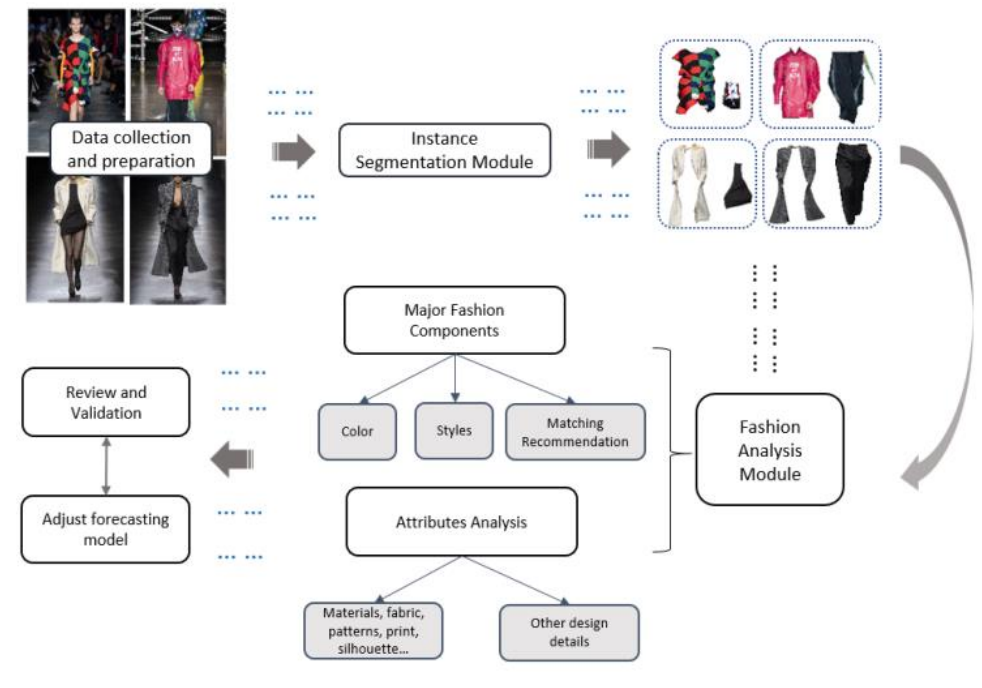

Although today's fashion trends research set up a bank of lots of resources such as catwalk shows, trunk shows, pop culture, street style, art, lifestyle and so on, catwalk remains as a crucial part of fashion delivery. Recent year, with the successful of deep learning algorithm in computer vision, especially in image classification and object detection, data-driven AI system using machine learning are used to analyze the complex images. With this in mind, our study aims to build a novel and 
cutting edge data-driven trend forecasting system to predict seasonal trends based on catwalk analysis (Named Neo-fashion system). Specifically, with a large scale catwalk data, our study (1) identifies objects and clothes on mannequin in images; (2) reveals three key components in trend forecasting including dominant colors, popular styles, and matching recommendations; (3) explores design details in terms of fabric, pattern, print, silhouette and so on; and (4) provides quantitative evidences and supports for fashion brands and companies to forecast seasonal trends.

In this study, we collected 32,702 catwalk images from WGSN catwalk database including four major seasonal collections - Spring/Summer, Autumn/Winter, Pre-Summer and Pre-Fall collection in 2019. After scratching the data from online database, we manage them into our cloud server database and then randomly select 2, 000 images to prepare labeling process. In total, 769 images were returned in our server with 23 major categories. In the Neo-Fashion system, we propose to use the state-of-the-art object detection algorithm in computer vision to detect fashion items only on the models and then use the cropped-out images of clothes to proceed analysis. Instance segmentation module and fashion trend analysis module were designed in the Neo-Fashion system. Figure 1 shows the proposed Neo-Fashion system to demonstrate the research flow of this study. Input catwalk images are given to instance segmentation module to generate mask of each fashion item. Then Fashion analysis module provides two parts of analysis, major fashion components and attributes analysis. Results from the previous stage are further combined to generate fashion trends. Finally, expert review and validation process are conducted to adjust forecasting model.
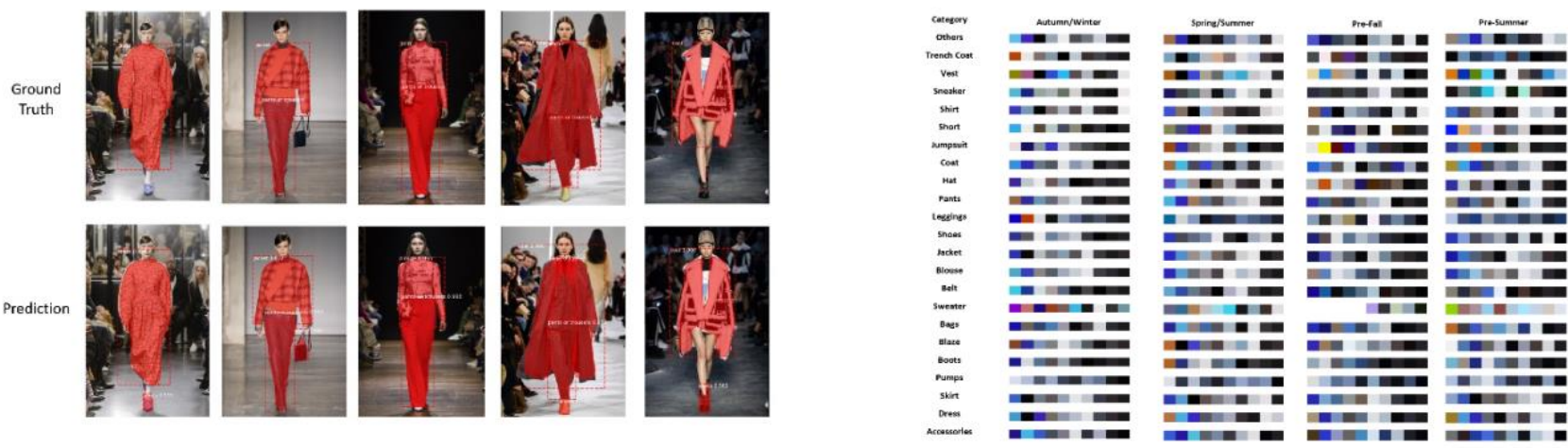

Figure 2 above shows the Illustration of instance segmented prediction in validation dataset. The upper images are labeled as ground truth while the bottom ones are results of prediction. Figure 3 above shows the Recommend Color Panel based on each Fashion Season. As shown in Fig 2, our mask prediction shows good results on recognizing regular cloth samples. In the 1st, 2nd and 5th column comparison, our learned model can predict the correct outline and classes of cloth. But there are still minor errors in prediction, like wrong classification and miss-recognized, as shown in 3rd and 4th column in Fig 2. In addition, our learned model is able to identify all the garments or accessories on fashion models, even not labeled by human coders. In Fig 3, top 10 dominant

Page 2 of 3

(c) 2020 The author(s). Published under a Creative Commons Attribution License (https://creativecommons.org/licenses/by/4.0/), which permits unrestricted use, distribution, and reproduction in any medium, provided the original work is properly cited.

ITAA Proceedings, \#77 - https://itaaonline.org 
colors, from right to left, are demonstrated. As shown in Fig 3, dominant colors in Spring/Summer are much brighter and lighter than Autumn/Winter which is consistent with the common sense. Some interesting findings were emerged too. With neutrals come back into favor, trend-driven colors were clearly identified.

In this paper, we proposed a novel data-driven fashion trend forecasting system, named NeoFashion. The system mainly focuses on catwalk image analysis by using machine learning algorithm which includes deep learning object detectors for instance segmentation, big data analysis and K-means clustering algorithm. Neo-fashion aims to provide supports for fashion researchers and practitioner to forecast trends. To best of our knowledge, this system is on the cutting-edge of AI system in fashion trend forecasting. For the future work, there are two directions for us to improve the performance of system. First, fine-grained classification needs to be implemented to improve the classification performance of instance segmentation module. Second, the prediction of attributes will be incorporated to advance trend forecasting in our NeoFashion system.

\section{References}

Bikhchandani, S., Hirshleifer, D., \& Welch, I. (1992). A theory of fads, fashion, custom, and cultural change as informational cascades. Journal of political Economy, 100(5), 992-1026.

Halland, G \& Jones, R (2017). Fashion Trend Forecasting. Laurence King Publishing. London. https://www.amazon.com/Fashion-Trend-Forecasting-GwynethHolland/dp/1786270587/ref=sr_1_1 ?keywords=trend+forecasting\&qid=1557524608\&s=g ateway\&sr $=8-1$

Han, X., Wu, Z., Jiang, Y. G., \& Davis, L. S. (2017, October). Learning fashion compatibility with bidirectional lstms. In Proceedings of the 25th ACM international conference on Multimedia, 1078-1086. ACM.

Liu, Z., Luo, P., Qiu, S., Wang, X., \& Tang, X. (2016). Deepfashion: Powering robust clothes recognition and retrieval with rich annotations. In Proceedings of the IEEE conference on computer vision and pattern recognition, 1096-1104.

Vittayakorn, S., Yamaguchi, K., Berg, A. C., \& Berg, T. L. (2015, January). Runway to realway: Visual analysis of fashion. In 2015 IEEE Winter Conference on Applications of Computer Vision, 951-958. IEEE.

Page 3 of 3

(c) 2020 The author(s). Published under a Creative Commons Attribution License (https://creativecommons.org/licenses/by/4.0/), which permits unrestricted use, distribution, and reproduction in any medium, provided the original work is properly cited.

ITAA Proceedings, \#77 - https://itaaonline.org 